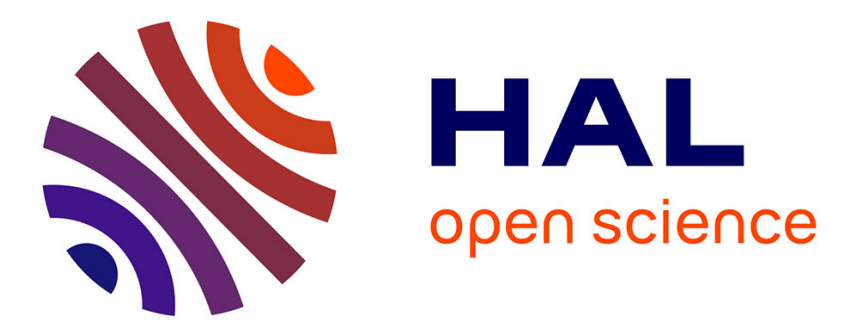

\title{
K-Player Bayesian Waterfilling Game for Fading Multiple Access Channels
}

He Gaoning, Merouane Debbah, Samson Lasaulce

\section{To cite this version:}

He Gaoning, Merouane Debbah, Samson Lasaulce. K-Player Bayesian Waterfilling Game for Fading Multiple Access Channels. 3rd IEEE International Workshop on Computational Advances in MultiSensor Adaptive Processing, Dec 2009, Netherlands. 4 p. hal-00446972

\section{HAL Id: hal-00446972 \\ https://hal-centralesupelec.archives-ouvertes.fr/hal-00446972}

Submitted on 13 Jan 2010

HAL is a multi-disciplinary open access archive for the deposit and dissemination of scientific research documents, whether they are published or not. The documents may come from teaching and research institutions in France or abroad, or from public or private research centers.
L'archive ouverte pluridisciplinaire HAL, est destinée au dépôt et à la diffusion de documents scientifiques de niveau recherche, publiés ou non, émanant des établissements d'enseignement et de recherche français ou étrangers, des laboratoires publics ou privés. 


\section{$K$-Player Bayesian Waterfilling Game for Fading Multiple Access Channels}

\author{
Gaoning $\mathrm{He}$ \\ Motorola Labs \& Télécom \\ ParisTech \\ 91193 Gif-sur-Yvette - FRANCE \\ gaoning.he@gmail.com
}

\author{
Mérouane Debbah \\ Alcatel-Lucent Chair in \\ Flexible Radio \& Supélec \\ 91193 Gif-sur-Yvette - FRANCE \\ merouane.debbah@supelec.fr
}

\author{
Eitan Altman \\ INRIA \\ 06902 Sophia Antipolis - FRANCE \\ eitan.altman@sophia.inria.fr
}

\begin{abstract}
We present a Bayesian game-theoretic approach for the distributed resource allocation problem in the context of $K$-user fading multiple access channels (MAC). We assume that users have incomplete information about the channel state information (CSI), i.e., each user knows his own channel state, but does not know the states of other users. All users (transmitters) are considered to be rational, selfish, and each one carries the objective of maximizing its own achievable data rate. In such a game-theoretic study, the central question is whether a Bayesian equilibrium (BE) exists. Based on the assumption of two channel states, we prove that there exists exactly one $\mathrm{BE}$ in this game.
\end{abstract}

\section{INTRODUCTION}

In recent years, there has been great interest in selforganizing wireless networks in which mobile devices allocate resource in a decentralized manner [1]. Tools of game theory [2] have been widely applied to study the resource allocation and power control problems in various types of networks, such as fading MAC [3], orthogonal frequency division multiplexing (OFDM) [4], multiple input and multiple output (MIMO) channels [5], [6], and interference channels [7], etc. Typically, the game-theoretic models used in these previous works assume that the information/knowledge about other devices is available to all devices. However, this assumption is hardly met in practice. In practical wireless communication scenarios, mobile devices can have local information but can barely access to global information on the network status.

In this paper, we focus our attentions on the fading MAC. The capacity region of fading MAC and the optimal resource allocation algorithms have been characterized and well studied in many pioneering works with assumptions of different information levels [8]-[11]. However, in order to achieve the full capacity region, it usually requires a central computing resource (a scheduler with comprehensive knowledge of the network information) to globally allocate the system resources. This process is centralized, it involves feedback and overhead communication whose load scales linearly with the number of transmitters and receivers in the network. In addition, with the fast evolution of wireless techniques, this centralized network infrastructure begins to expose its weakness in many aspects, e.g., slow reconfiguration against varying environment, increased computational complexity, etc.

A static non-cooperative game has been introduced in the context of fading MAC, known as "waterfilling game" [3].
From a simple two-user MAC setting, the authors show that the unique Nash equilibrium (NE) [12] of the corresponding waterfilling game is actually the maximum sum-rate point on the boundary of the MAC capacity region. However, their results rely on the fact that both transmitters have "complete information" about the CSI, and in particular, perfect CSI of all transmitters in the network. As we previously pointed out, this assumption is rarely possible in practice.

Thus, this power allocation game needs to be reconstructed with some realistic assumptions made on the knowledge level of mobile devices. Under this consideration, it is of great interest to investigate scenarios in which devices have "incomplete information" about their components, e.g., a device is ware of its own channel gain, but unaware of the channel gains of other devices. In game theory, a strategic game with incomplete information is called a "Bayesian game". Over the last ten years, Bayesian game-theoretic tools have been used to design distributed resource allocation strategies only in a few contexts, e.g., CDMA networks [13], [14], multicarrier interference networks [15]. Our motivation is therefore to study how Bayesian games can be applied to the context of fading MAC. The goal of this paper is to investigate the outcome of our Bayesian waterfilling game, i.e., the existence and uniqueness of BE point(s).

The paper is organized in the following form: In Section $\amalg$ we introduce the system model and describe briefly the background. In Section III] the $K$-user MAC is formulated as a Bayesian game. In Section IV we characterize the BE set. Finally, we close with some concluding remarks in Section $\nabla$

\section{System Model AND BACKGROUND}

\section{A. System Model}

We consider a time-slotted flat-fading MAC in a singlecell network, in which $K$ users are simultaneously sending information to one base station. At time $t$, the signal received by the base station can be mathematically expressed as

$$
y(t)=\sum_{k=1}^{K} \sqrt{g_{k}(t)} x_{k}(t)+z(t)
$$

where $x_{k}(t)$ and $g_{k}(t)$ are the input signal and fading channel gain of user $k, z(t)$ is a zero-mean white Gaussian noise with 
variance $\sigma^{2}$. The input signal $x_{k}(t)$ can be further written as

$$
x_{k}(t)=\sqrt{p_{k}(t)} s_{k}(t)
$$

where $p_{k}(t)$ and $s_{k}(t)$ are the transmitted power and data of user $k$ at time $t$.

We assume that the channel gains $g_{1}, \ldots, g_{K}$ are deterministic constants during the period of each transmission block (which is assumed to be larger than a time slot interval). Therefore, within each time slot $t$, this is simply a Gaussian multi-user channel [16]. Now, instead of considering the whole capacity region, we are interested in the single-user achievable rate (assuming that the base station uses low complexity single-user decoder [16]), i.e.,

$$
R_{k}=\log \left(1+\frac{g_{k} p_{k}}{\sigma^{2}+\sum_{j=1, j \neq k}^{K} g_{j} p_{j}}\right)
$$

Intuitively, the object of each user is to maximize this rate, which represents the amount of transmitted information.

\section{B. Complete and Incomplete Information}

Before introducing the problem, it is necessary to clarify the meanings of complete information and incomplete information. In game theory, the notion of complete information means that all players know completely the structure of the game, which includes:

- Player set (how many devices involved? what are they?)

- Other players' actions (what are their behaviors?)

- Other players' payoffs (what are their object functions?)

If some players do not completely know the game structure, we call it a game with incomplete information. For example, "chess" is a game with complete information, because the chess moves are known to all players; "poker" is a game with incomplete information, because players are uncertain about the card states of other players, therefore, the exact object functions of other players are unknown.

In our context, take an example of two-user MAC, complete information means that the channel gains $g_{1}, g_{2}$ are available at both transmitters $\mathrm{Tx}_{1}, \mathrm{Tx}_{2}$; incomplete information means that the channel gain $g_{k}$ is only available at $\operatorname{Tx}_{k}, k=1,2$. In this paper, we assume that the player set and action set (see their definitions in Section III) are known to all players.

\section{Background: Waterfilling Game}

An important related work is the "waterfilling game" [3], where multi-user resource allocation problem in fading MAC is studied in detail as a non-cooperative game with complete information. In this game, users are assumed to compete with transmission rates as payoff and transmit powers as moves.

Here, we briefly describe the problem, which is also helpful to understand our Bayesian game formulation. Under the assumption of complete information, each user $k$ 's transmit power strategy can be considered as a function of all users' channel gains, i.e., $p_{k}\left(g_{1}, \ldots, g_{K}\right)$. In the case of two-user $\mathrm{MAC}$, for a fixed strategy $p_{2}\left(g_{1}, g_{2}\right)$, finding the optimal strategy $p_{1}\left(g_{1}, g_{2}\right)$ for user 1 requires solving the following optimization problem:

$$
\begin{aligned}
\max _{p_{k}} & \mathbb{E}_{g_{1}, g_{2}}\left[\log \left(1+\frac{g_{1} p_{1}\left(g_{1}, g_{2}\right)}{\sigma^{2}+g_{2} p_{2}\left(g_{1}, g_{2}\right)}\right)\right] \\
\text { s.t. } & \mathbb{E}_{g_{1}, g_{2}}\left[p_{1}\left(g_{1}, g_{2}\right)\right] \leq P_{1}^{\max } \\
& p_{1}\left(g_{1}, g_{2}\right) \geq 0
\end{aligned}
$$

and similarly for user 2 . Here, $P_{1}^{\max }$ is the average power constraint of user 1 . Note that the solution of (2) depends on user 2's strategy $p_{2}(\cdot)$, which user 1 does not know, and reciprocally for user 2 . However, the static non-cooperative game model given in [3] enables both users to adjust their strategies adaptively to their guesses of the strategy of the other user. This process converges to a unique NE:

$$
\left\{\begin{array}{l}
p_{1}^{\star}\left(g_{1}, g_{2}\right)=\left(\lambda_{1}-\frac{\sigma^{2}}{g_{1}}\right)^{+}, \text {when } g_{1} \geq \frac{\lambda_{2}}{\lambda_{1}} g_{2} \\
p_{2}^{\star}\left(g_{1}, g_{2}\right)=\left(\lambda_{2}-\frac{\sigma^{2}}{g_{2}}\right)^{+}, \text {when } g_{2} \geq \frac{\lambda_{1}}{\lambda_{2}} g_{1}
\end{array}\right.
$$

with $p_{1}^{\star}\left(g_{1}, g_{2}\right)=0$ and $p_{2}^{\star}\left(g_{1}, g_{2}\right)=0$ in all other cases. The dual variables $\lambda_{1}$ and $\lambda_{2}$ are given by

$$
\left\{\begin{array}{l}
\mathbb{E}_{g_{1}, g_{2}}\left[\left(\lambda_{1}-\frac{\sigma^{2}}{g_{1}}\right)^{+} \mid g_{1} \geq \frac{\lambda_{2}}{\lambda_{1}} g_{2}\right]=P_{1}^{\max } \\
\mathbb{E}_{g_{1}, g_{2}}\left[\left(\lambda_{2}-\frac{\sigma^{2}}{g_{2}}\right)^{+} \mid g_{2} \geq \frac{\lambda_{1}}{\lambda_{2}} g_{1}\right]=P_{2}^{\max }
\end{array}\right.
$$

The authors show that the NE (3) corresponds to the maximum sum-rate point of the capacity region. This result is somewhat surprising, since NE is in general inefficient comparing to the Pareto optimality. Thus, an interesting conclusion is that the selfish behavior of the users leads them to jointly optimize the sum-rate of the channel.

\section{BAYESIAN GAME MODEL}

Before introducing our game model, we need to clarify a prior assumption, as follows,

Assumption III.1. We assume that each user's channel gain $g_{k}$ is i.i.d. from two discrete values: $g_{-}$and $g_{+}$with probability $\rho_{-}$and $\rho_{+}$, respectively. WloG, we assume $g_{-}<g_{+}$.

On the one hand, our assumption is closely related to the way how feedback information are signalled to the transmitters. In order to get the channel information $g_{k}$ at the transmitter side, it requires the base station to estimate it and then feedback to user $k$ at a given precision. Since in digital communications any information is represented by a finite number of bits (e.g., $x$ bits), channels gains are mapped into a set that contains a finite number of states ( $2^{x}$ states).

On the other hand, this is a necessary assumption for analytical tractability, since in principle the functional strategic form of a player can be quite complex with both actions and states are continuous (or infinite). To avoid this problem, in [15] the authors successfully modelled a multi-carrier Gaussian interference channel as a Bayesian game with discrete (or finite) actions and continuous states. Inspired from [15], in this paper, we model the fading MAC as a Bayesian game under the assumption of continuous actions and discrete states. 
Now, we model the $K$-user fading MAC as a Bayesian game. In such a communication system in which users have incomplete information, the natural object of each user is to maximize its average achievable rate, i.e.,

$$
\begin{array}{ll}
\max _{p_{k}} & \mathbb{E}_{\mathbf{g}}\left[\log \left(1+\frac{g_{k} p_{k}\left(g_{k}\right)}{\sigma^{2}+\sum_{j=1, j \neq k}^{K} g_{j} p_{j}\left(g_{j}\right)}\right)\right] \\
\text { s.t. } & \mathbb{E}_{g_{k}}\left[p_{k}\left(g_{k}\right)\right] \leq P_{k}^{\max } \\
& p_{k}\left(g_{k}\right) \geq 0
\end{array}
$$

where $\mathbf{g}=\left\{g_{1}, \ldots, g_{K}\right\}$ is a set of channel gains, $P_{k}^{\max }$ is the average power constraint for user $k$. Note that under the assumption that each user has only incomplete information about the fading channel gains, user $k$ 's power strategy $p_{k}(\cdot)$ can only rely on its own channel gain $g_{k}$, written as $p_{k}\left(g_{k}\right)$.

For a given strategy $p_{-k}=\left\{p_{1}, \ldots, p_{k-1}, p_{k+1}, \ldots, p_{K}\right\}$, the single-user maximization problem (5) is a convex optimization problem [17]. Via Lagrangian duality, the solution is given by the following equation:

$$
\mathbb{E}_{g_{-k}}\left[\frac{g_{k}}{\sigma^{2}+g_{k} p_{k}\left(g_{k}\right)+\sum_{j \neq k} g_{j} p_{j}\left(g_{j}\right)}\right]=\lambda_{k}
$$

where $g_{-k}=\left\{g_{1}, \ldots, g_{k-1}, g_{k+1}, \ldots, g_{K}\right\}$, and the dual variable $\lambda_{k}$ is chosen such that the power constraint in (5) is satisfied with equality. However, the solution of (6) also depends on $p_{-k}(\cdot)$ which user $k$ does not know. Thus, in order to obtain the optimal power allocation, each user must adjusts its power level based on the guess of all other users' strategies. Given the following game model, each user is able to adjust its strategy according to the belief it has on the strategy of the other user.

The $K$-player MAC Bayesian waterfilling game can be completely characterized as:

$$
\mathcal{G}_{M A C} \triangleq\langle\mathcal{K}, \mathcal{T}, \mathcal{P}, \mathcal{Q}, \mathcal{U}\rangle
$$

- Player set: $\mathcal{K}=\{1, \ldots, K\}$.

- Type set: $\mathcal{T}=\mathcal{T}_{1} \times \ldots \times \mathcal{T}_{K}$ (' $\times$ ' is the Cartesian product) where $\mathcal{T}_{k}=\left\{g_{-}, g_{+}\right\}$, a player's type is defined as its channel gain, i.e., $g_{k} \in \mathcal{T}_{k}$.

- Action set: $\mathcal{P}=\mathcal{P}_{1} \times \ldots \times \mathcal{P}_{K}$ where $\mathcal{P}_{k}=\left[0, P_{k}^{\max }\right]$, a player's action is defined as its transmit power, i.e., $p_{k} \in \mathcal{P}_{k}$.

- Probability set: $\mathcal{Q}=\mathcal{Q}_{1} \times \ldots \times \mathcal{Q}_{K}$ where $\mathcal{Q}_{k}=\left\{\rho_{-}, \rho_{+}\right\}$, we have $\rho_{+}=\operatorname{Pr}\left(g_{k}=g_{+}\right)$and $\rho_{-}=\operatorname{Pr}\left(g_{k}=g_{-}\right)$.

- Payoff set: $\mathcal{U}=\left\{u_{1}, \ldots, u_{K}\right\}$ where $u_{k}$ is chosen as player $k$ 's achievable rate (1)

$$
u_{k}\left(p_{1}, \ldots, p_{K}\right)=\log \left(1+\frac{g_{k} p_{k}\left(g_{k}\right)}{\sigma^{2}+\sum_{j=1, j \neq k}^{K} g_{j} p_{j}\left(g_{j}\right)}\right)
$$

In games of incomplete information, a player's type represents any kind of private information that is relevant to its decision making. In our context, the fading channel gain $g_{k}$ is naturally considered as the type of user $k$ 's, since its decision (in terms of transmit power) can only rely on $g_{k}$.

\section{BAYESIAN EQUILIBRIUM}

What we can expect of the outcome from a Bayesian game? Generally speaking, the process of rational players' behaviors usually results in BE, which represents a "stable" result of learning and evolution of all participants. Therefore, it is important to characterize its set, since it concerns the performance analysis of a distributed system.

\section{A. Definition of Bayesian Equilibrium}

Let $\left\{\hat{p}_{k}(\cdot), p_{-k}(\cdot)\right\}$ denote the strategy profile where all players play $p(\cdot)$ except player $k$ who plays $\hat{p}_{k}(\cdot)$, we can then describe player $k$ 's payoff as:

$$
u_{k}\left(\hat{p}_{k}, p_{-k}\right)=u_{k}\left(p_{1}, \ldots, p_{k-1}, \hat{p}_{k}, p_{k+1}, \ldots, p_{K}\right)
$$

\section{Definition IV.1. (Bayesian equilibrium)}

The strategy profile $p^{\star}(\cdot)=\left\{p_{k}^{\star}(\cdot)\right\}_{k \in \mathcal{K}}$ is a (pure strategy) Bayesian equilibrium, if for all $k \in \mathcal{K}$, and for all $p_{k}(\cdot) \in \mathcal{P}_{k}$ and $p_{-k}(\cdot) \in \mathcal{P}_{-k}$

$$
\bar{u}_{k}\left(p_{k}^{\star}, p_{-k}^{\star}\right) \geq \bar{u}_{k}\left(p_{k}, p_{-k}^{\star}\right)
$$

where we define $\bar{u}_{k} \triangleq \mathbb{E}_{\mathbf{g}}\left[u_{k}\right]$.

From this definition, it is clear that at the BE no player can benefit by changing its strategy while the other players keep theirs unchanged. It is worth to mention that the action set of each player is independent of the type set, i.e., the actions available to user $k$ is the same for every its type.

\section{B. Characterization of Bayesian Equilibrium Set}

It is well known that, in general, an equilibrium point does not necessarily exist [2]. Therefore, our primary interest is to investigate the existence and uniqueness of $\mathrm{BE}$ in $\mathcal{G}_{M A C}$. Here, we directly give our main result:

Theorem IV.2. There exists a unique Bayesian equilibrium in the K-user MAC game $\mathcal{G}_{M A C}$.

Proof: It is easy to prove the existence part, since the strategy space $p_{k}$ is convex, compact and nonempty for each $k$; the payoff function $u_{k}$ is continuous in both $p_{k}$ and $p_{-k}$; and $u_{k}$ is concave in $p_{k}$ for any $p_{-k}$ [2].

In order to prove the uniqueness part, we should rely on a sufficient condition [18]: a non-cooperative game has a unique equilibrium, if the non-negative weighted sum of the payoffs is diagonally strictly concave. We firstly give the definition:

Definition IV.3. (Diagonally strictly concave)

Function $f(\mathbf{x}, \mathbf{r})=\sum_{i=1}^{n} r_{i} \varphi_{i}(\mathbf{x})$ is called diagonally strictly concave for any vector $\mathbf{x} \in \mathbb{R}^{n \times 1}$ and fixed vector $\mathbf{r} \in \mathbb{R}_{++}^{n \times 1}$, if for any two different vectors $\mathbf{x}^{0}, \mathbf{x}^{1}$, we have

$$
\Omega\left(\mathbf{x}^{0}, \mathbf{x}^{1}, \mathbf{r}\right) \triangleq\left(\mathbf{x}^{1}-\mathbf{x}^{0}\right)^{\mathrm{T}} \delta\left(\mathbf{x}^{0}, \mathbf{r}\right)+\left(\mathbf{x}^{0}-\mathbf{x}^{1}\right)^{\mathrm{T}} \delta\left(\mathbf{x}^{1}, \mathbf{r}\right)>0
$$

where $\delta(\mathbf{x}, \mathbf{r})$ is called pseudo-gradient of $f(\mathbf{x}, \mathbf{r})$, defined as

$$
\delta(\mathbf{x}, \mathbf{r}) \triangleq\left[\begin{array}{c}
r_{1} \frac{\partial \varphi_{1}}{\partial x_{1}} \\
\vdots \\
r_{n} \frac{\partial \varphi_{n}}{\partial x_{n}}
\end{array}\right] .
$$


Lemma IV.4. The weighted non-negative sum of the average payoffs $\bar{u}_{k}$ in $\mathcal{G}_{M A C}$ is diagonally strictly concave.

Proof: Write the sum of the average payoffs as:

$$
f^{u}(\mathbf{p}, \mathbf{r}) \triangleq \sum_{k=1}^{K} r_{k} \bar{u}_{k}(\mathbf{p}),
$$

where $\mathbf{p}=\left[p_{1} \ldots p_{K}\right]^{\mathrm{T}}$ is the transmit power vector, $\mathbf{r}=\left[r_{1} \ldots r_{K}\right]^{\mathrm{T}}$ is a non-negative vector assigning weights $r_{1}, \ldots, r_{K}$ to the average payoffs $\bar{u}_{1}, \ldots, \bar{u}_{K}$, respectively. Similar to 9), we let $\delta^{u}(\mathbf{p}, \mathbf{r}) \triangleq\left[r_{1} \frac{\partial \bar{u}_{1}}{\partial p_{1}} \ldots r_{K} \frac{\partial \bar{u}_{K}}{\partial p_{K}}\right]^{\mathrm{T}}$ be the pseudo-gradient of $f^{u}(\mathbf{p}, \mathbf{r})$. Now, we define

$$
p_{k} \triangleq p_{k}\left(g_{-}\right), k \in \mathcal{K}
$$

the transmit power of player $k$ when its channel gain is $g_{-}$. Since we have shown from Lagrangian that at the equilibrium the power constraint is satisfied with equality, i.e., $\sum_{g_{k}} p_{k}\left(g_{k}\right)=P_{k}^{\max }$, we have $P_{k}^{\max }-p_{k}=p_{k}\left(g_{+}\right) k \in \mathcal{K}$, as the transmit power when its channel gain is $g_{+}$. Therefore, it is easy to find that the average payoff $\bar{u}_{k}$ can be actually transformed into a weighted sum-log function, as follows

$$
\bar{u}_{k}\left(p_{k}\right)=\sum_{i} \omega_{i} \log \left[1+\frac{\alpha_{k}^{i}+\beta_{k}^{i} p_{k}}{\sigma^{2}+\sum_{j \neq k}\left(\alpha_{j}^{i}+\beta_{j}^{i} p_{j}\right)}\right]
$$

where $i$ represents the index for different jointly probability events, $\omega_{i}$ represents the corresponding probability for index $i$. Note that the following conditions hold for all $i, k$

$$
\alpha_{k}^{i}+\beta_{k}^{i} p_{k} \geq 0, \alpha_{k}^{i}>0, \beta_{k}^{i} \neq 0, \sigma^{2}>0
$$

Now, we can write the pseudo-gradient $\delta^{u}$ as

$$
\delta^{u}(\mathbf{p}, \mathbf{r})=\left[\begin{array}{c}
r_{1} \frac{\partial u_{1}}{\partial p_{1}} \\
\vdots \\
r_{K} \frac{\partial u_{K}}{\partial p_{K}}
\end{array}\right]=\left[\begin{array}{c}
r_{1} \sum_{i} \beta_{1}^{i} \phi_{i}^{-1}(\mathbf{p}) \\
\vdots \\
r_{K} \sum_{i} \beta_{K}^{i} \phi_{i}^{-1}(\mathbf{p})
\end{array}\right]
$$

where function $\phi_{i}(\mathbf{x})$ is defined as

$$
\phi_{i}(\mathbf{x}) \triangleq \sigma^{2}+\sum_{k=1}^{K}\left(\alpha_{k}^{i}+\beta_{k}^{i} x_{k}\right)
$$

To check the diagonally strictly concave condition (8), we let $\mathbf{p}^{0}, \mathbf{p}^{1}$ be two different vectors, and define

$$
\begin{aligned}
\Omega^{u}\left(\mathbf{p}^{0}, \mathbf{p}^{1}, \mathbf{r}\right) & \triangleq\left(\mathbf{p}^{1}-\mathbf{p}^{0}\right)^{\mathrm{T}} \delta^{u}\left(\mathbf{p}^{0}, \mathbf{r}\right)+\left(\mathbf{p}^{0}-\mathbf{p}^{1}\right)^{\mathrm{T}} \delta^{u}\left(\mathbf{p}^{1}, \mathbf{r}\right) \\
& =\sum_{i}\left[\phi_{i}^{-1}\left(\mathbf{p}^{0}\right)-\phi_{i}^{-1}\left(\mathbf{p}^{1}\right)\right] \zeta_{i} \\
& =\sum_{i} \phi_{i}^{-1}\left(\mathbf{p}^{\mathbf{0}}\right) \phi_{i}^{-1}\left(\mathbf{p}^{\mathbf{1}}\right) \zeta_{i}^{2}
\end{aligned}
$$

where $\zeta_{i}=\sum_{k=1}^{K} r_{k} \beta_{k}^{i}\left(p_{k}^{1}-p_{k}^{0}\right)$. Define $\Delta p_{k}=p_{k}^{1}-p_{k}^{0}$, since $\mathbf{p}^{0}, \mathbf{p}^{1}$ are two different vectors, we must have $\Delta \mathbf{p}=$ $\left[\Delta p_{1} \cdots \Delta p_{K}\right]^{\mathrm{T}} \neq \mathbf{0}$. Now, we can draw a conclusion from the equation above: $\Omega^{u}\left(\mathbf{p}^{0}, \mathbf{p}^{1}, \mathbf{r}\right)>0$. This is because: (1) the first part $\phi_{i}^{-1}\left(\mathbf{p}^{0}\right) \phi_{i}^{-1}\left(\mathbf{p}^{\mathbf{1}}\right)>0$ for all $i$, since $\sigma^{2}>0$ and $\alpha_{k}^{i}+\beta_{k}^{i} p_{k} \geq 0$ for all $i, k$; (2) the second part $\zeta_{i}^{2} \geq$ 0 for all $i$, and there exists at least one nonzero term $\zeta_{i}^{2}$, due to $\Delta \mathbf{p} \neq 0$ and $r_{k} \neq 0, \beta_{k}^{i} \neq 0$ for all $i, k$. Therefore, the summation of all the products of the first and the second terms must be positive. From Definition IV.3, the sum-payoff function $f^{u}(\mathbf{p}, \mathbf{r})$ satisfies the condition of diagonally strictly concave. This completes the proof of this lemma.

Since our sum-payoff function $f^{u}(\mathbf{p}, \mathbf{r})$ 10 is diagonally strictly concave, from the Theorem 2 in [18], we have the uniqueness of Nash equilibrium in our game $\mathcal{G}_{M A C}$.

\section{CONCLUSION}

We presented a Bayesian game-theoretic framework for distributed resource allocation in fading MAC, where users are assumed to have only local information about the network channel gain states. By introducing the assumption of finite channel states, we successfully found a analytical way to characterize the BE set. We proved the existence and uniqueness of BE in our game. This result is important for predicting the system performance of a distributed wireless network.

\section{REFERENCES}

[1] M. Debbah, "Mobile Flexible Networks: The challenges ahead", 2008 International Conference on Advanced Technologies for Communications, invited paper, Hanoi, Vietnam.

[2] D. Fudenberg and J. Tirole, Game Theory, MIT Press, 1991.

[3] L. Lai and H. El Gamal, "The Water-Filling Game in Fading MultipleAccess Channels", IEEE Trans. on Info. Theory, Vol. 54, No. 5, pp. 2110-2122, May 2008.

[4] G. He, S. Beta and M. Debbah, "Game-Theoretic Deployment Design of Small-Cell OFDM Networks", 3rd ICST/ACM International Workshop on Game Theory in Communication Networks, Oct. 2009.

[5] S. Lasaulce, M. Debbah and E. Altman, "Methodologies for analyzing equilibria in wireless games", submitted to IEEE Signal Processing Magazine, Special issue on Game Theory for Signal Processing, 2009.

[6] E. V. Belmega, S. Lasaulce, and M. Debbah, "Power Allocation Games for MIMO Multiple Access Channels with Coordination", IEEE Trans. on Wireless Communications, revised, Sept. 2008.

[7] E. Altman, M. Debbah and A. Silva, "Game Theoretic Approach for Routing in Dense Ad-hoc Networks", Stochastic Networks Workshop, Edinburg, UK, July, 2007.

[8] R. Cheng and S. Verdú, "Gaussian Multiaccess Channels with ISI: Capacity Region and Multiuser Water-filling", IEEE Tran. on Info. Theory, vol. 39, May 1993.

[9] R. Knopp and P. A. Humblet, "Information Capacity and Power Control in Single-cell Multiuser Communications", presented at the International Conference on Communications, Seattle, WA, June 1995.

[10] A. Goldsmith and P. Varaiya, "Capacity of Fading Channel with Channel Side Information”, IEEE Trans. on Info. Theory, vol. 43, pp. 1986-1992, Nov. 1995.

[11] D. Tse and S. Hanly, "Multi-access Fading Channels Part I: Polymatroid Structure, Optimal Resource Allocation and Throughput Capacities", IEEE Trans. on Info. Theory, vol. 44, no. 7, pp. 2796-2815, Nov. 1998.

[12] J. F. Nash, "Equilibrium Points in N-points Games", Proc. of the National Academy of Science, vol. 36, no. 1, pp. 48-49, Jan. 1950.

[13] T. Heikkinen, "A Minmax Game of Power Control in a Wireless Network under Incomplete Information” DIMACS, Tech. Rep. 99-43, Aug. 1999.

[14] S. Jean and B. Jabbari, "Bayesian Game-theoretic Modeling of Transmit Power Determination in a Self-organizing CDMA Wireless Network", Proc. IEEE VTC Fall 2004, vol. 5, pp. 3496-3500, Sep. 2004.

[15] S. Adlakha, R. Johari and A. Goldsmith, "Competition in Wireless Systems via Bayesian Interference Games", http://arxiv.org/abs/0709.0516. Sep. 2007.

[16] T. M. Cover and J. A. Thomas, Elements of Information Theory, Wiley, 1991.

[17] S. Boyd and L. Vandenberghe, "Convex Optimization," Cambridge University Press, 2004.

[18] J. B. Rosen, "Existence and Uniqueness of Equilibrium Points for Concave N-person Games," Econometrica, vol. 33, pp. 520-534, July 1965. 